Solar Energy Materials \& Solar Cells 76 (2003) 599 - 611; presented on NATO Advanced Study Institute, Sozopol, Bulgaria, $10^{\text {th }}-22^{\text {nd }}$ September 2001

\title{
CELLO: an advanced LBIC measurement technique for solar cell local characterization
}

\author{
J. Carstensen*, G. Popkirov, J. Bahr, and H. Föll \\ Faculty of Engineering, Christian-Albrechts University of Kiel, Kaiserstr. 2, D-24143 Kiel, Germany
}

\begin{abstract}
An advanced LBIC measurement for solar cell local characterization, called CELLO has been developed and tested on mono- and multi-crystalline Si solar cells. A solar cell is illuminated with near to $1.5 \mathrm{AM}$ light intensity and additionally subjected to an intensity modulated and scanning local illumination by a focused IR-laser. The linear response (current or potential) of the solar cell is measured for various fixed global conditions (different preset voltage or current values) during scanning. A large number of independent data with high spatial resolution are obtained. Applying an advanced fitting procedure on these data yields a set of local parameters for each point on the solar cell which give information on the spatial distribution of the photo current, the series and shunt resistance, the lateral diffusion of minority carriers, the quality of the back surface field and even allows the calculation of local IV-curves. The theoretical and experimental approach to this technique will be discussed and the applicability of this new solar cells characterization tool will be demonstrated.
\end{abstract}

\section{Introduction}

A solar cell is a large area device, thus its global IV-characteristic and efficiency strongly depend on local properties. The existence of local defects, such as locally decreased diffusion length, strong local shunt- or high local series resistances, may adversely influence the solar cell global properties. Experimental techniques suitable to map the spatial distribution of such local parameters can provide valuable information, and thus help to improve the technology for production of efficient and reproducible solar cells.

The LBIC (Light Beam Induced Current) is a well known technique for mapping of the spatial distribution of the photo current of a solar cell [1-5]. LBIC is usually employed under short-circuit current conditions and allows the calculation of the local diffusion length of the solar cell material from local photo current data. The mapping analyzer PVScan 5000 by NREL [6] can be used to map defects and grain boundaries using reflectivity data, and special surface etching, and minority carriers diffusion length using LBIC measurement with correction for surface reflectivity. Localized shunts can be mapped by sensitive infrared CCD-cameras [7, 8] or nematic liquid

\footnotetext{
${ }^{*}$ Corresponding author.
} 
crystal thermography [9]. Electron-beam-induced current (EBIC) is an alternative technique for the investigation of point and extended defects in semiconductors and solar cells [10]. A destructive technique called MASC (Mesa diode Analysis of Solar Cells) was developed recently [11] for local IV-characterization of a solar cell and mapping of the open circuit voltage, and the fill-factor. Another destructive technique for resistance analysis by mapping of potential (RAMP) [12] is based on the measurement of the potential between the back of the solar cell and its top surface by a scanning (scratching) tungsten electrode. Approaches to map the local variation of resistivity through thin film solar cells have been reported recently [13, 14]. The authors used scanning local laser illumination at various intensities and spot sizes without bias illumination. It was shown, that the respective local material resistivity can be extracted from the parameters that best fit to the measured photocurrent data.

The aim of this paper is to describe a new advanced LBIC measurement technique, called 'CELLO', which is, to the best of our knowledge, the first tool that allows the determination of all local parameters on large area silicon solar cells, especially the local series- and shunt resistance, $R_{s}(x, y)$ and $R_{s h}(x, y)$, and thus to identify all materialand process-induced, efficiency relevant defects. In principle, the data obtained could also be used to simulate the behavior of the complete solar cell for any set of technology parameters.

\section{The measurement technique}

A simplified schematic diagram of CELLO is depicted in Fig. 1. The solar cell is illuminated homogeneously by a set of halogen lamps with near to $1.5 \mathrm{AM}$ intensity. Additionally, a sinusoidally modulated infrared laser beam is focused onto the sample through a piezo-controlled mirror and provides a X-Y-scanned local perturbation. A potentiostat/galvanostat is used to pre-set the voltage (potentiostatic control) or the current (galvanostatic control) of the solar cell. A lock-in amplifier, synchronized to the laser beam modulation signal, is used to measure the solar cell response to the laser beam perturbation - a.c. current or voltage, respectively.

The CELLO technique essentially measures the global response of a solar cell to local perturbations for several pre-set working points of the cell as shown in Fig. 2. CELLO works in the linear regime by analyzing the small signal response of the solar cell. Several sets of data for $d I\left(V_{\text {cell }}, x, y\right)$ and $d V\left(I_{\text {cell }}, x, y\right)$ are measured for pre-set constant values of $V_{\text {cell }}$ or $I_{\text {cell }}$, according to Fig. 2. The amplitude of the modulated laser beam can be expressed as a current $d I_{p h}$ (the photo current signal on the solar cell for infinite diffusion length and neglectable recombination at the back surface) and the ratios $d I / d I_{p h}\left(V_{\text {cell }}, x, y\right)$ and $d V / d I_{p h}\left(I_{\text {cell }}, x, y\right)$ represent local transfer functions. The data obtained are fitted to a complete (and partially novel) model of the solar cell which allows one:

- to draw solar cell surface maps of the measured data,

- to calculate maps of the local series- and shunt resistances, diffusion length and back-surface field,

- to construct the complete local IV-curve for each point of the solar cell.

In this context, the well-known LBIC mode is just the measurement of $d I / d I_{p h}(0, x, y)$. 


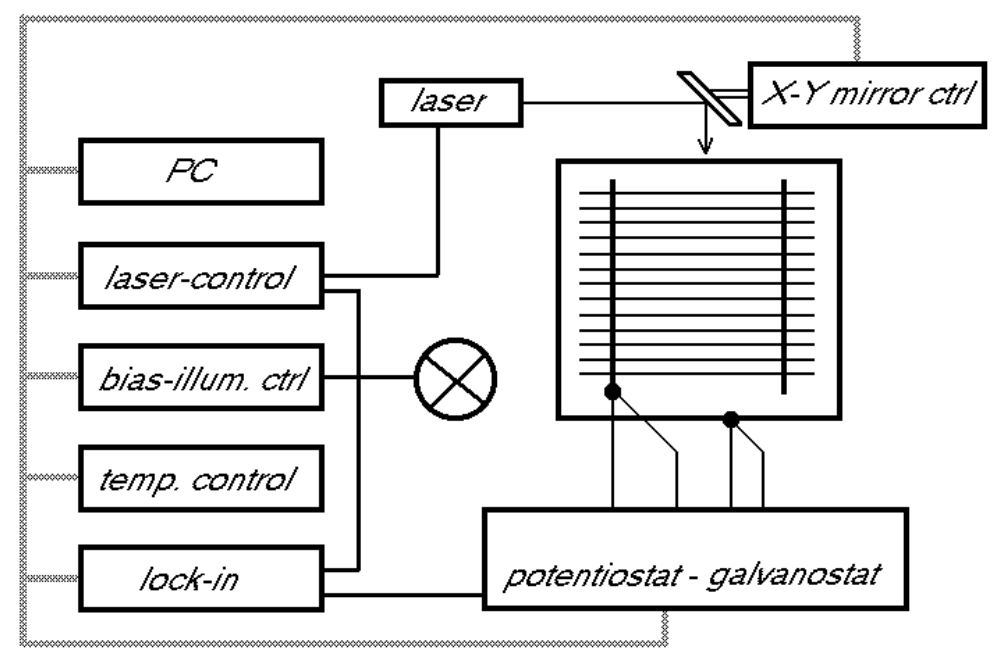

Fig. 1: The CELLO system

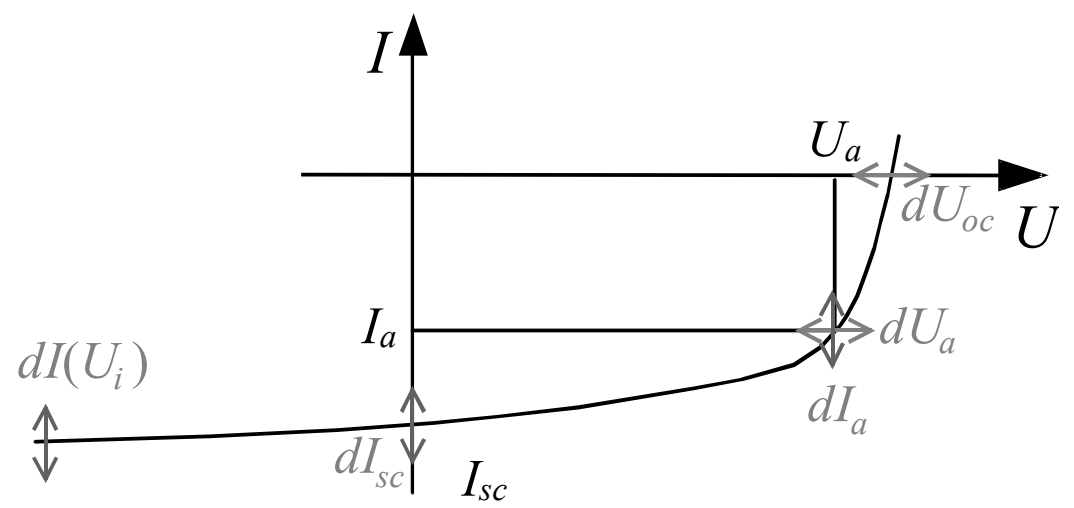

Fig. 2: At several points along the IV curve of a solar cell the linear response of the current (potentiostatic control) or of the voltage (galvanostatic control) to an additional local illumination is measured.

During a CELLO measurement of a $100 \mathrm{~mm}$ x $100 \mathrm{~mm}$ solar cell about 350.000 points are scanned in several modes, leading to nearly two million data points, collected. Thus, measurement time is an issue. The $\mathrm{X}-\mathrm{Y}$ piezo-controlled mirror provides fast positioning of the laser beam spot, thus the integration time of the lock-in amplifier which defines among other factors the $\mathrm{S} / \mathrm{N}$ ratio, remains as a major limiting factor. Although, the integration time needed for a constant $\mathrm{S} / \mathrm{N}$ ratio decreases with increasing modulation frequency of the laser, the useable frequency range is limited ( $\leq$ $1 \mathrm{kHz}$ ) by the capacitance of the $p n$-junction of the solar cell. Thus, some technical problems had to be overcome in order to shorten measurement time at acceptable $\mathrm{S} / \mathrm{N}$ ratio. The laser beam intensity must be set low enough in order to assure linear response. Thus, for example, the a.c. photo currents due to the modulated laser illumination are in the range of $100 \mu \mathrm{A}$. On the other hand, the d.c. global cell currents at the maximum power point with bias illumination can exceed $1 \mathrm{~A}$. Therefore, the intensity of the global illumination has to be extremely constant and noise-free during the measurement. The bias illumination has to be also homogeneous over the whole area of the solar cell. Since very small (series) resistance's are to be determined, it is 
essential to provide good contacts to the cell. Thus, separate wires for current supply and voltage measurement have been used to connect the solar cell to the potentiostat. A home-made, computer-controlled, low-noise potentiostat/galvanostat is used for voltage/current control and current measurement with high $\mathrm{S} / \mathrm{N}$ ratio. The system realized so far measures about 30 points per second. The laser beam is focussed to about $100 \mu \mathrm{m}$ diameter. The potentiostat allows for 3 A current with a stability of some $\mu \mathrm{A}(\mu \mathrm{V})$ in a frequency range up to $10 \mathrm{kHz}$.

\section{Modeling and calculations}

Assuming that sufficient data sets have been obtained experimentally, the raw data must be converted into local parameters of the solar cell. This is done with the help of the equivalent circuit diagram as shown in Fig. 3. The solar cell is divided in a global part (denoted "complete solar cell" on the left) and a local part (on the right) which are distinguished by different sets of parameters for their respective elements. Since we are looking locally at a very small part of the solar cell (the laser beam spot), the global part can be described by a constant set of parameters and is known by measuring the IV curve. To a sufficiently good approximation, any local part (with a locally changing set of parameters) can be added without changing the global values. In a conventional equivalent circuit diagram both parts would be connected via one resistor; but this proved not to be sufficient for the present task. There are two important modifications in the equivalent circuit shown in Fig. 3 with respect to conventional diagrams:

- the two parts of the cell are connected via two resistors as shown,

- the combination of the local diode and the local current source (modeling the photo current induced by the laser) is not described by the usual equations, but couples the diode current, $I_{d}$, and the photo-currents $I_{p h}$ and $I_{p h, 0}$, respectively, via

$$
I_{p h}=I_{p h, 0}-C_{r e c} I_{d}^{3} \text {. }
$$

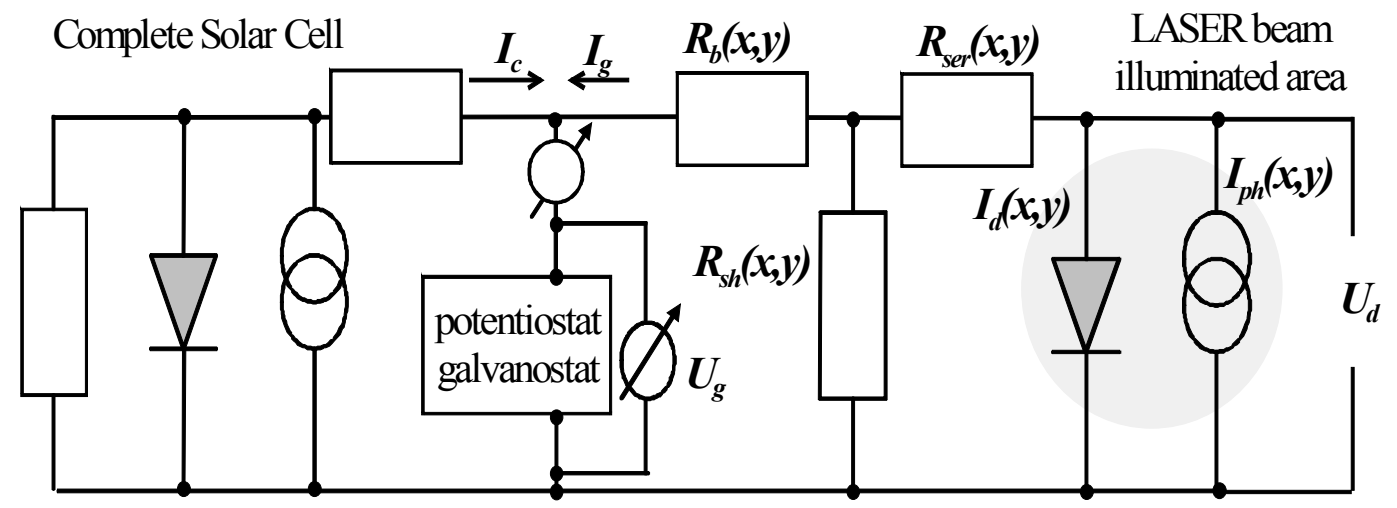

Fig. 3: Equivalent circuit for the interpretation and evaluation of the CELLO measurements. The gray area connecting the local diode and the local illumination source marks the coupling between these elements, being the source of the additional losses as described by Eq. (1). 
Here $I_{p h, 0}$ and $I_{p h}$ are the generated and the collected photo currents for a given point of the solar cell surface, respectively. $C_{r e c} I_{d}^{3}$ represents the current due to local recombination losses with $C_{r e c}$ as a fit parameter. This equation essentially describes the lateral diffusion and recombination of minority carriers and expresses a sensitivity of the solar cell to gradients of the diffusion length. The impetus for this modification has sound physical reasons, but stems primarily from the impossibility to fit the experimental data on multicrystalline solar cells with its strong lateral gradients in the diffusion length to a simpler diagram. In fact, not all carriers, photo generated close to the surface are collected and contribute to the current at finite values of the voltage. Some carriers are needed to generate the voltage and these carriers diffuse in the bulk and thus experience recombination in areas laterally removed from their respective point of origin. If the local diffusion length varies significantly in the lateral direction, this will influence the local properties of the point under consideration. A mathematical analysis of this effect leads to the (simplified) Eq. (1). Taking into account the lateral diffusion length variation by Eq. (1) leads to almost perfect fits of the experimental data obtained. As a general result, it follows that the average diffusion length of a solar cell material is not a sufficient measure of its quality. The gradients in the diffusion length distribution are just as important.

The complete set of equations (under the assumption that the grid is an equipotential surface) is

$$
\begin{aligned}
& I_{g}=I_{d}+I_{s h}-I_{p h} \\
& I_{d}=I_{d 1}\left(e^{\frac{q U_{d}}{n_{1} k T}}-1\right)+I_{d 2}\left(e^{\frac{q U_{d}}{n_{2} k T}}-1\right) \\
& U_{g}=R_{s h} I_{s h}+R_{b} I_{g}
\end{aligned}
$$

Here, $k$ is the Boltzmann constant, $T$ is the temperature, $q$ is the electron charge, $I_{d l}$, $n_{1}$ and $I_{d 2}, n_{2}$ are the saturation currents and ideality factors of the two diodes respectively. $R_{b}$ is the lateral resistance of the emitter between the illuminated 'pixel' and the grid. Photo carriers generated at one point can be collected by the grid, or lost through a local shunt or by recombination. Using approximations to linear order in the local photo current, induced by the laser beam, this set of equations is generally sufficient to extract all required quantities by fitting the experimental data. The absolute values obtained, as well as the calculated local IV-curves, differ strongly for 'good' and 'bad' areas of the solar cell. CELLO measurements are extremely sensitive to local defects in the solar cell. The complete equivalent circuit diagram and therefore the complete set of local characteristics can be calculated from a finite set of experimental data. However, it takes many hours to measure the many sets of data needed, thus, a special routine has been developed that allows identification of areas of interest with only one measurement. After tedious transformations of Eq. (1) - (4) using some minor simplifications, an important analytical result for current measurements in forward bias emerges: 


$$
\frac{\frac{d I_{g}(U=0)}{d I_{g}(U)}-1}{\frac{\partial I_{c}}{\partial U_{c}}(U)}=R_{s e r} \frac{\frac{\partial I_{d}}{\partial U_{d}}(U)}{\frac{\partial I_{c}}{\partial U_{c}}(U)}\left(1+3 C_{r e c} I_{d}^{2}\right) \approx R_{\text {ser }}
$$

The parameters on the left side are directly measurable: $d I_{g}(U=0)$ is the standard LBIC current; $d I_{g}(U)$ is the current response, preferably at the optimal working point of the solar cell, defined by $U$, and $M I_{c} / M U_{c}(U)$ is the slope of the IV-curve of the complete solar cell at the working point. The plot of Eq. (5) indicates areas with nontrivial deviant behavior with respect to the series resistance $R_{s e r}$, the local diode $I_{d}$ current and/or increased local recombination of minority carriers due to lateral gradients in the diffusion length distribution (indicated by $C_{r e c}$ ). Thus, this plot marks the areas with possible defects where further measurements could be fruitful. Since for many homogeneous solar cells the relation

$$
\frac{\frac{\partial I_{d}}{\partial U_{d}}(U)}{\frac{\partial I_{c}}{\partial U_{c}}(U)}\left(1+3 C_{r e c} I_{d}^{2}\right) \approx 1
$$

holds, i.e. the slope of the local IV-curve is comparable with that of the global IVcurve and no enhanced lateral recombination processes occur, a plot of the left-hand side of Eq. (5) yields directly the series resistance of the solar cell. Rewriting Eq. (5) we get

$$
\frac{d I_{g}(U=0)}{d I_{g}(U)}-1=R_{s e r} \frac{\partial I_{d}}{\partial U_{d}}(U)\left(1+3 C_{r e c} I_{d}^{2}\right)
$$

So, according to Eq. (7) the simple function

$$
F(A, B, x, y)=\left(\frac{d I_{A}(x, y)}{d I_{B}(x, y)}-1\right) * 1000
$$

contains information on the local series resistance, the diode quality, and additional recombination losses. Here $A$ and $B$ denote two different points of the IV-characteristic where measurements of $d I$ have been made (cf. Fig.2). For example, taking $U_{A}=-250$ $\mathrm{mV}$ (reverse bias condition) and $U_{B}=0 \mathrm{mV}$ (short-circuit conditions as in LBIC-mode) the map of $F$ is even sensitive to the local shunt resistance which corresponds to a bad local diode quality.

Like the LBIC technique, CELLO can measure the short circuit current locally, but it will be very difficult to measure the reflectivity of the wafer with CELLO, therefore we do not get information about the quantum efficiency. All other measurements are analyzed relatively to, e.g. the short circuit current. So for all other parameters local differences in the reflectivity are not important. Local IV-curves can be calculated using data for the local shunt and series resistances, and the local diffusion length. 


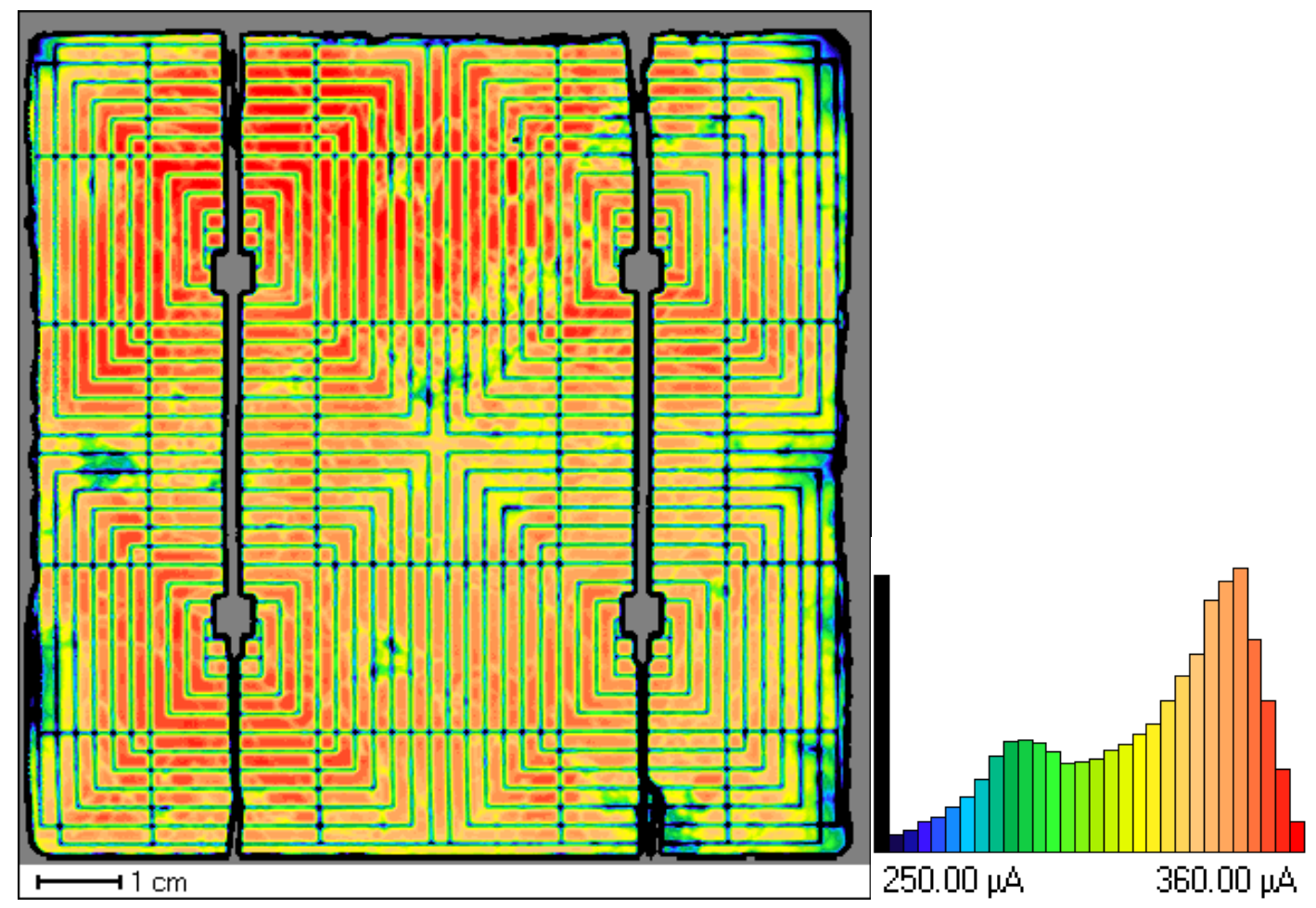

Fig. 4: Map of the linear current response $d I_{l}$ obtained potentiostatically at $U=-250 \mathrm{mV}$.

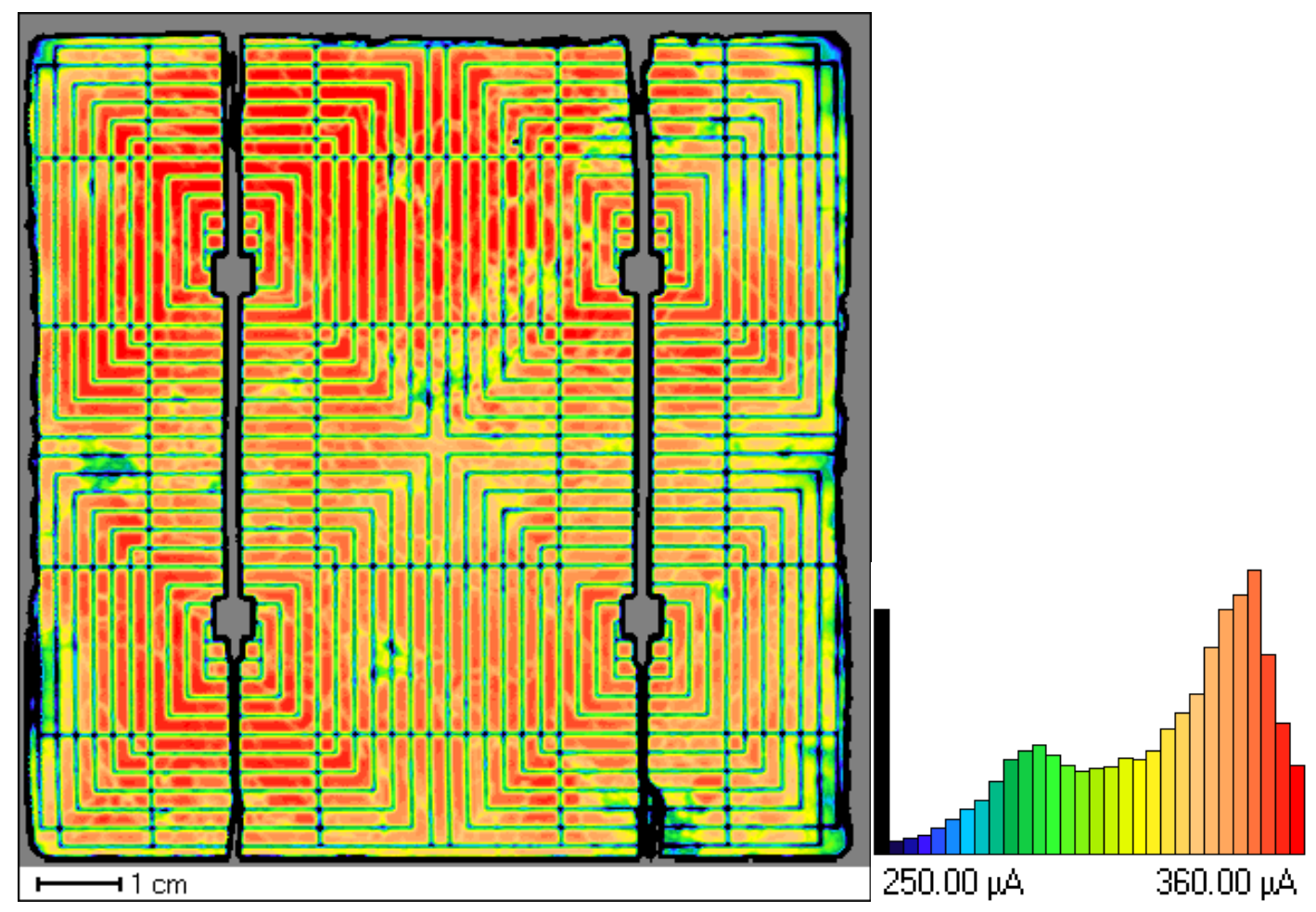

Fig. 5: Map of the linear current response $d I_{2}$, obtained potentiostatically at $U=0 \mathrm{mV}$. 


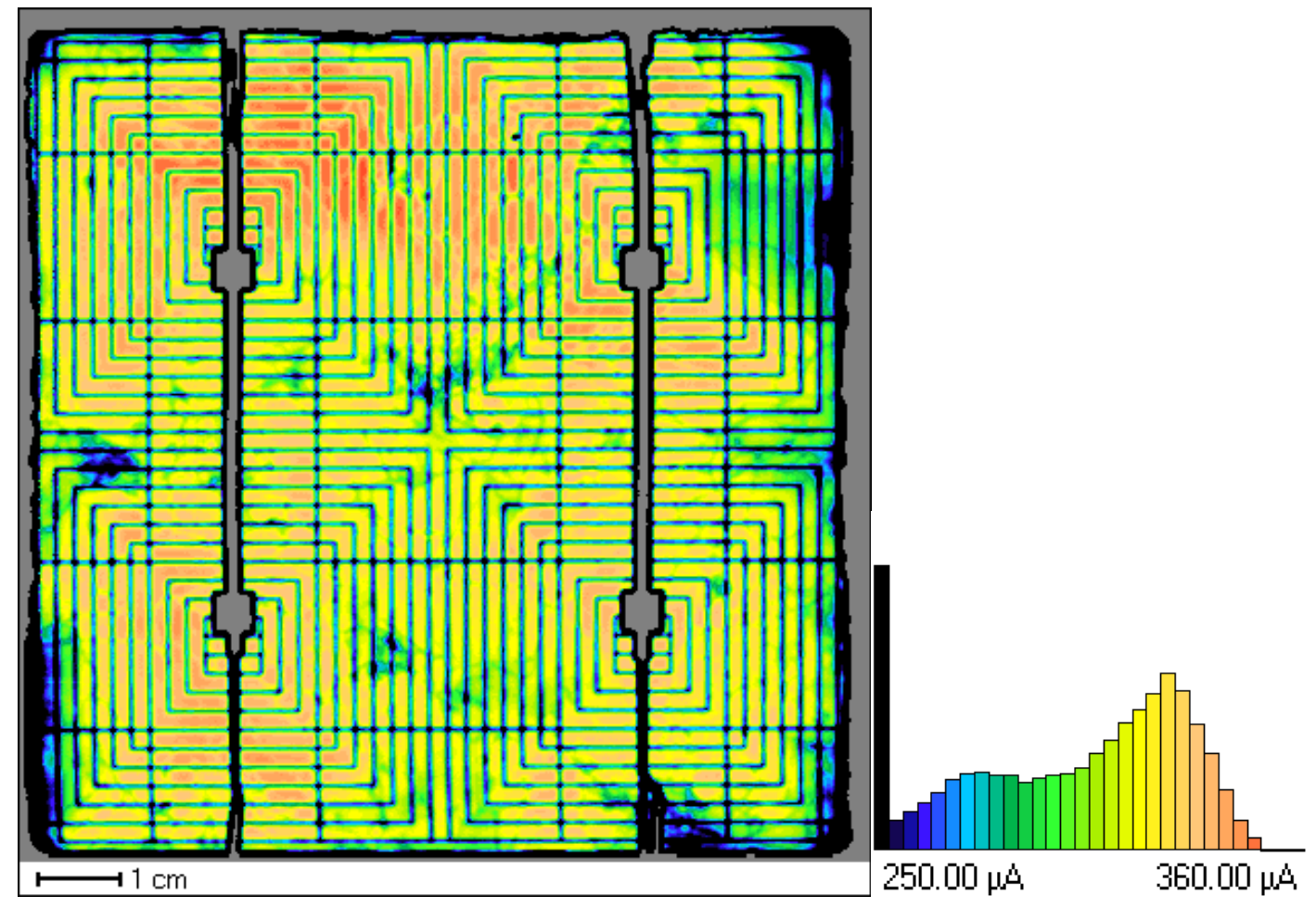

Fig. 6: Map of the linear current response $d I_{3}$, obtained potentiostatically at $U=300 \mathrm{mV}$.

\section{Results}

The CELLO equipment and its control program were repeatedly tested with various silicon $p n$-junction solar cells and are currently extensively used to test solar cells from different producer. The operation of CELLO will be illustrated here by the series of maps which can be obtained from one measurement on a solar cell, made on multicrystalline silicon wafer. First, direct maps of raw data, according to Fig. 2, will be presented in order to demonstrate how the different cell defects can alter the expected (homogeneous) surface distribution of the current (or voltage) small signal response to the laser beam perturbation. Maps of the linear current responses $d I_{1}, d I_{2}$, and $d I_{3}$, obtained potentiostatically at $U=-250 \mathrm{mV}, 0 \mathrm{mV}$ and $300 \mathrm{mV}$ are depicted in Figs. 4, 5 , and 6 , respectively. The maps of the voltage responses $d U_{1}$ and $d U_{2}$, measured galvanostatically at fixed cell currents $I=100 \mathrm{~mA}$ and $300 \mathrm{~mA}$ are shown in Fig. 7 and Fig.8. A multicrystalline solar cell with a grid design allowing for a very homogeneous collection of photo generated carriers has been chosen to illustrate several aspects of the CELLO analysis. On the first glance the three current maps (Figs. 4-6) do not look significantly different. The forward bias map (cf. Fig. 6) shows a slightly reduced current response in comparison to the short circuit condition as may be expected in correspondence to the global IV curve. Eq. (8) used to analyze the relative differences $F(1,2, x, y)$ between $d I_{1}$ and $d I_{2}$ (cf. Fig.9) yields a structure, which according to the CELLO theory can be related to ohmic shunts on the solar cell (dark areas in Fig. 9). This was proved by comparison of such maps with IR thermography [8]. Obviously, the solar cell under test has many edge shunts and even a large number of volume shunts. The changes in Fig. 9 between regions with and without shunts are in the per mill range. 


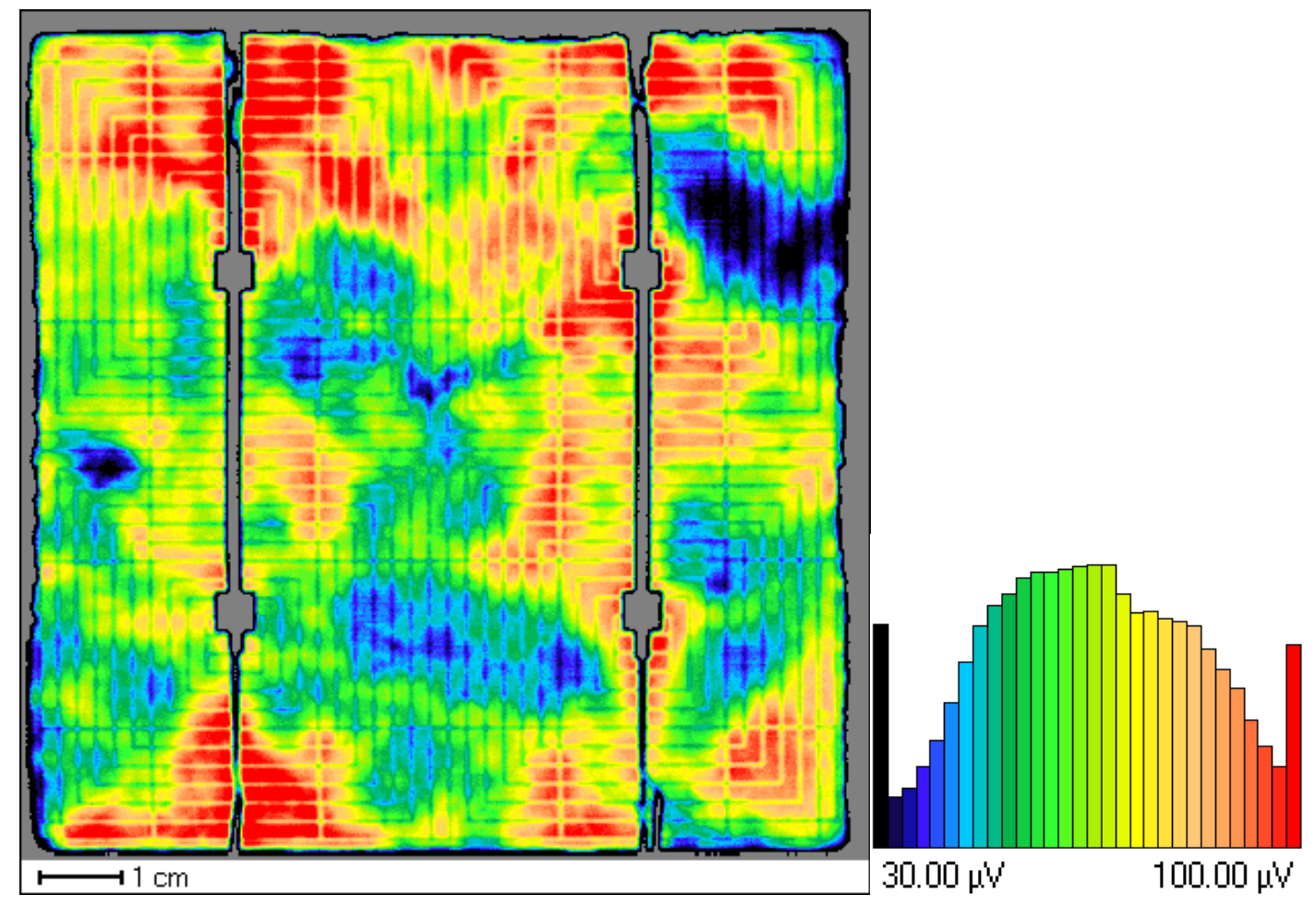

Fig. 7: Map of the voltage response $d U_{l}$, measured galvanostatically at fixed cell current $I=100 \mathrm{~mA}$.

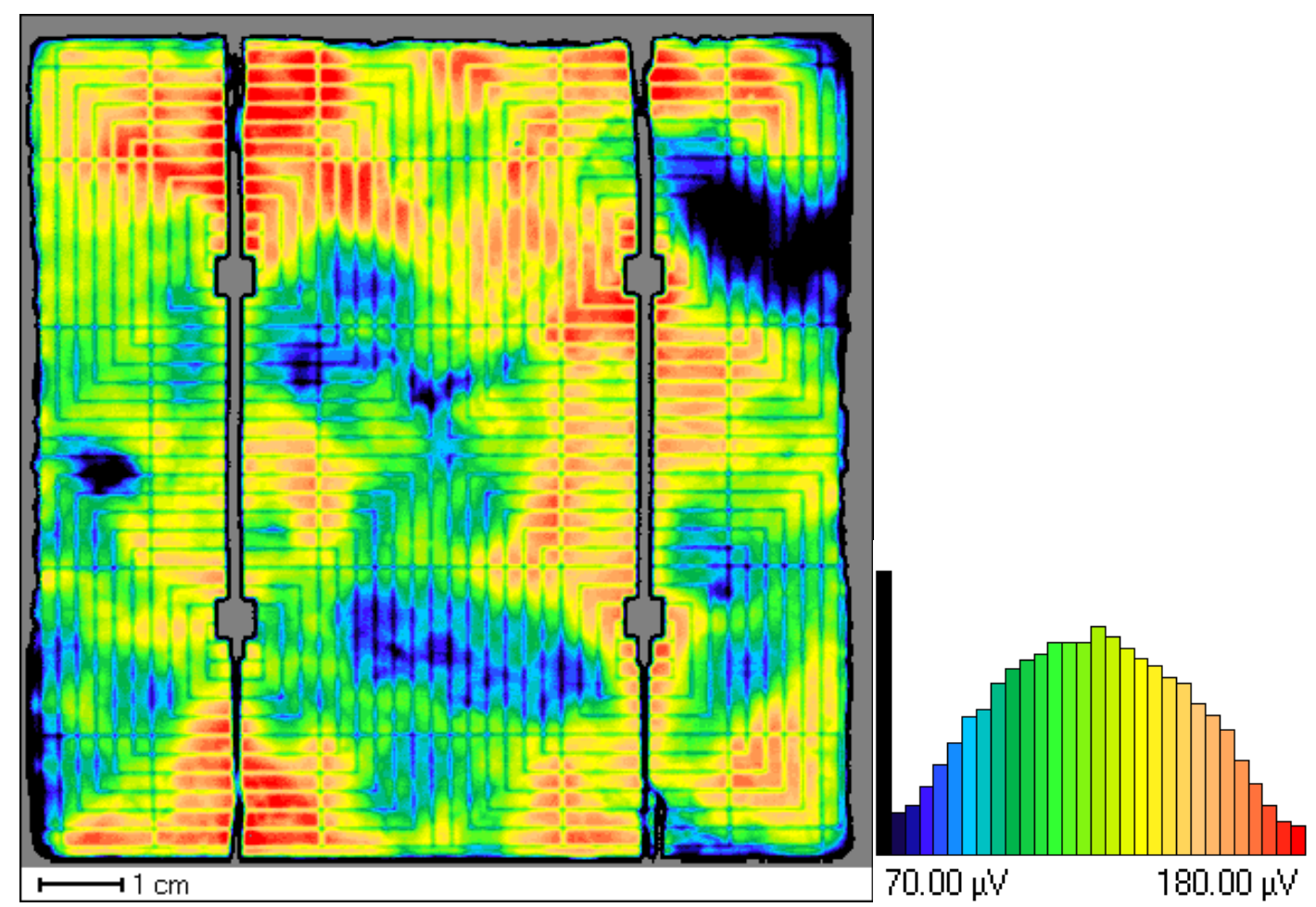

Fig. 8: Map of the voltage response $d U_{2}$, measured galvanostatically at fixed cell currents $I=300 \mathrm{~mA}$ 


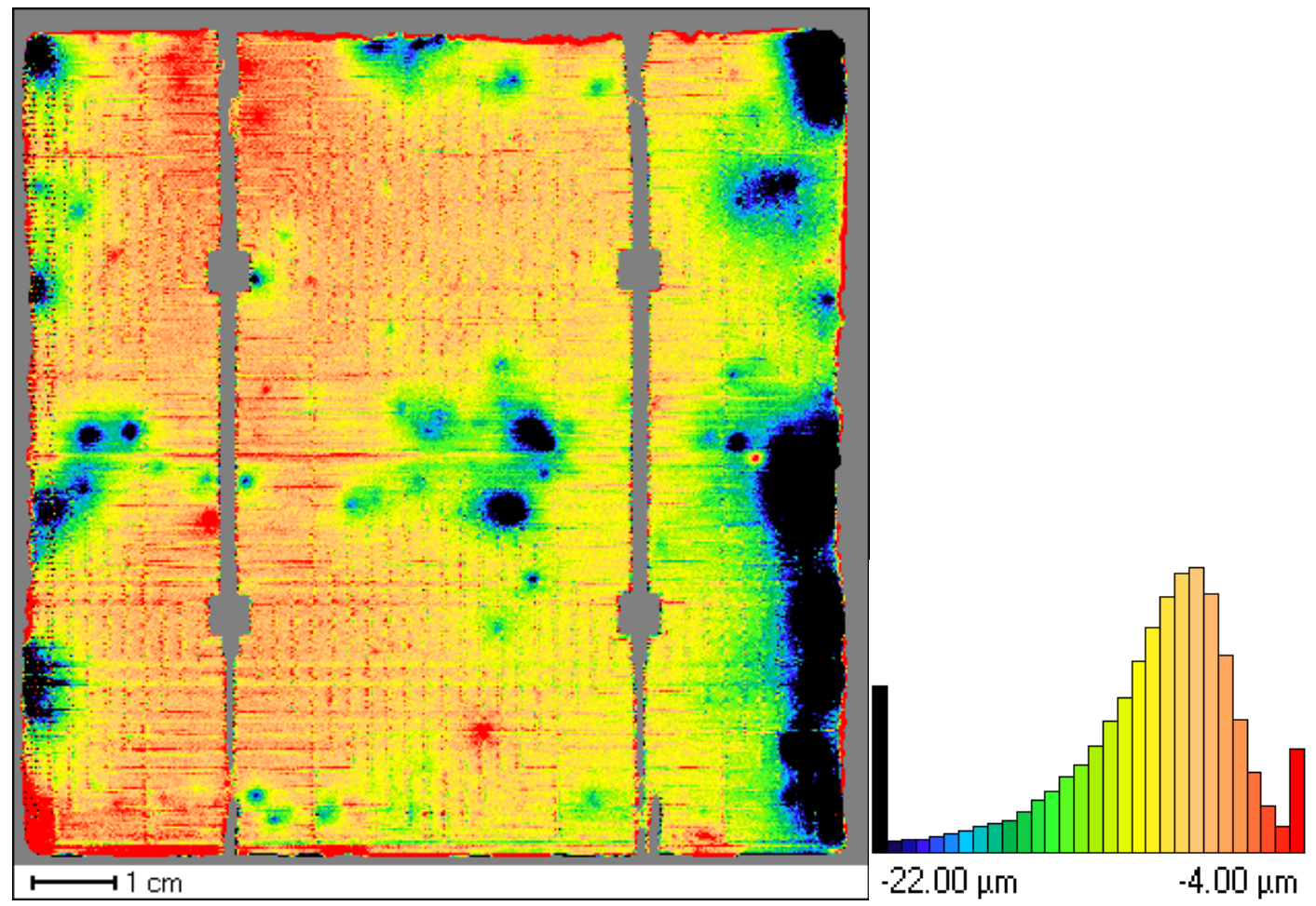

Fig. 9: Map of $F(1,2, x, y)=\left(d I_{1} / d I_{2}-1\right) * 1000$, which can be related to the ohmic shunts on the solar cell (the dark areas).

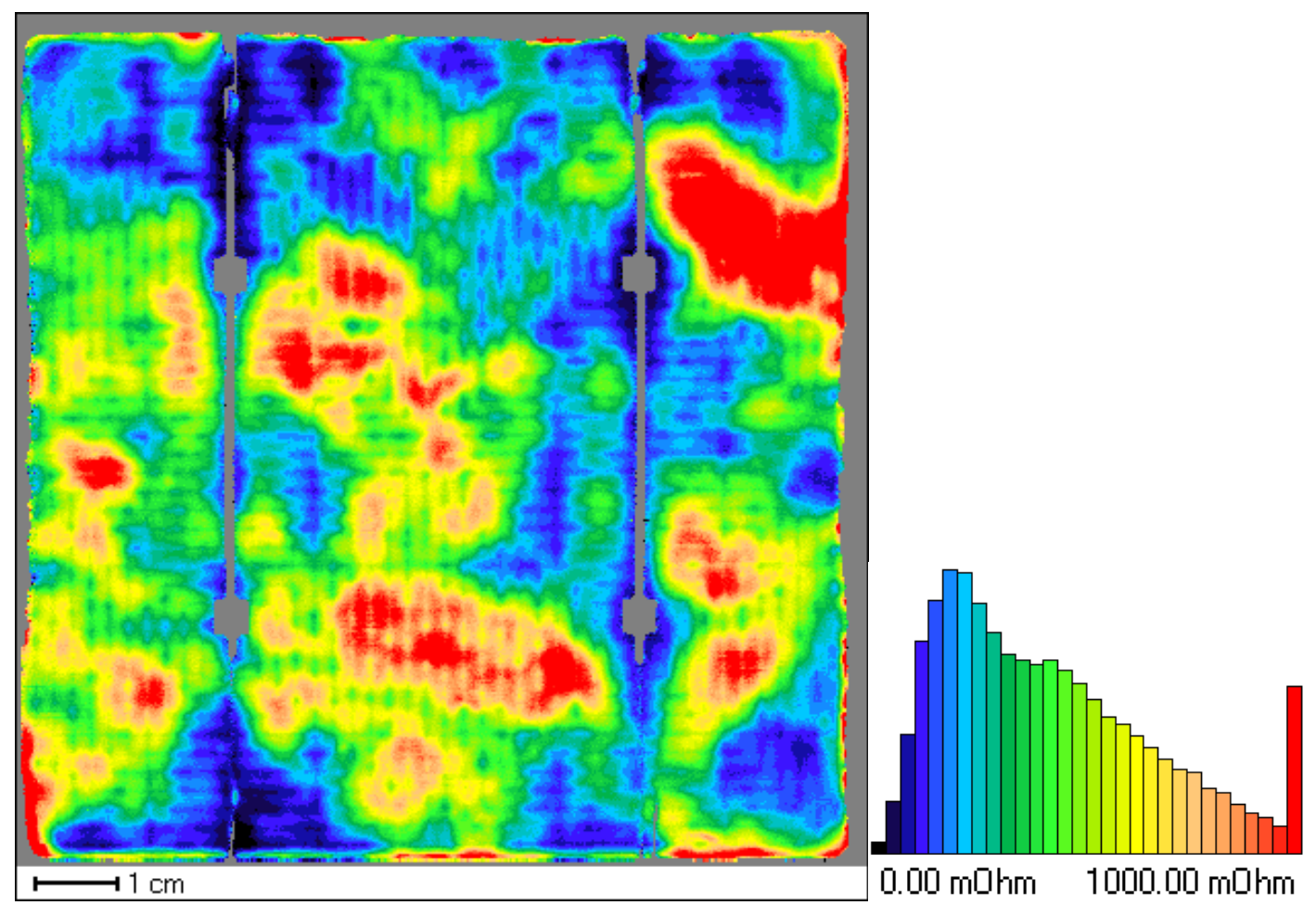

Fig. 10: Map of the effective series resistance $R_{\text {ser }}$. 


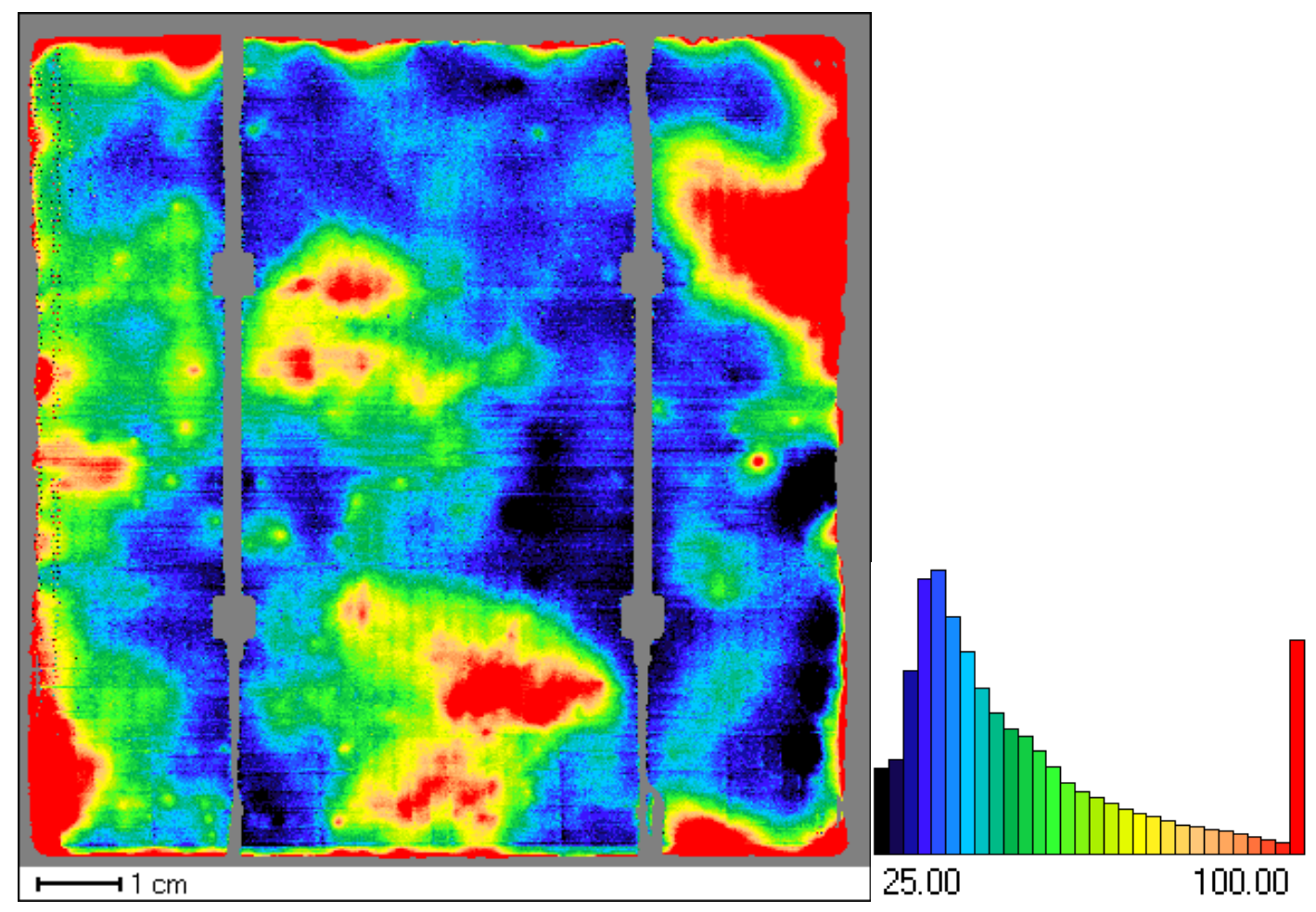

Fig. 11: Map of $\left(d I_{1} / d I_{3}-1\right) * 1000$.

Despite of the fact that the laser induced signal is extremely small it has to be measured with high precision in order to be sensitive to shunts. The relatively low sensitivity to shunts of the CELLO maps do not mean that shunts are not relevant.

Only the response of a shunt to the local illumination is quite weak since it depends on the fraction $R_{s h} / R_{s e r}$ between the shunt and the series resistance. Therefore, for quantifying the shunt resistance one has to determine the local series resistance as well.

Choosing a galvanostatic working point near open circuit condition, the linear response of the voltage reflects the ohmic losses from the illuminated point to the contacts of the solar cell. A small value (dark areas in Fig. 7) corresponds to strong ohmic losses. Again, these areas are neither correlated to the photo current maps nor to the shunt map. A further analysis which is beyond the scope of this article shows that in the solar cell under test the reduced voltage response originates from inhomogeneities in the emitter- and grid resistance. By compensating for the differences in the collected photo current, a map of the effective series ohmic resistance can be calculated (cf. Fig. $10)$.

Near the working point of the solar cell a mixture of the three relevant parameters: photo current, shunts, and series resistance will add to the linear response, combined additionally with the local diode characteristics. By calculating $F(1,3, x, y)$ we get rid of the influence of the photo current, as shown in Fig. 11. The resulting map reflects many structures of the series resistance as should be expected. Since $d I_{3}$ and $d U_{2}$ are measured at the same working point, the local diode characteristic $\frac{\partial I_{d}}{\partial U_{d}}(U)$ as well as the additional recombination losses have exactly the same value for both maps at each point. Thus we find nearly the same structures in the map $d U_{2} / d I_{1}$ in Fig. 12 as in Fig. 11. 


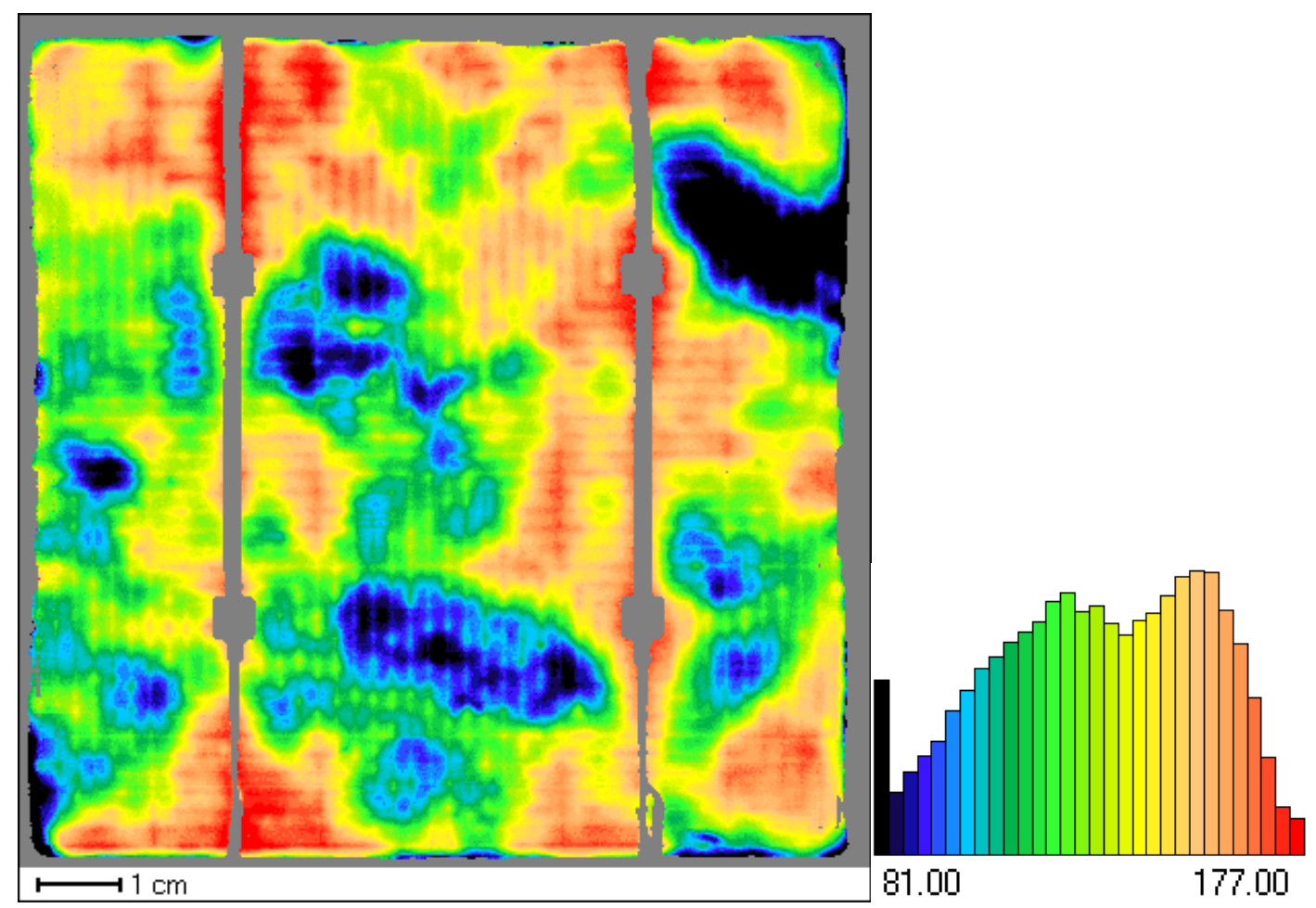

Fig. 12: Map of $d U_{2} / d I_{1}$ (in arbitrary units).

To compensate again for the differences in the local diffusion length not $d U_{2}$ but the ratio $d U_{2} / d I_{1}$ is plotted. Fig. 11 and 12 show structures visible in none of the other maps. These structures are most probably directly related to the local diode characteristics $\frac{\partial I_{d}}{\partial U_{d}}(U)$, but the presented data is not sufficient for a reliable identification of the type of defects at such areas.

Measuring two complete curves $d I(U)$ and $d U(I)$ in a relevant area and fitting these curves to the complete model allows to extract detailed information on all local solar cell parameters. This has been done for many defect types and the detailed results will be presented elsewhere.

\section{Conclusions}

These first results demonstrate that CELLO is a universal method for detecting and characterizing local defects in all solar cells since it is not restricted to silicon or crystalline materials. Including CELLO results into a detailed simulation program for solar cells, should provide a powerful tool for improving the efficiency of solar cells since this would allow to systematically optimize the technology for particular materials and processes. 


\section{References}

[1] C. Donolato, Solid State Electron., 68 (1982).

[2] J. Marek, J. Appl. Phys. 55 (1984) 2.

[3] J.F. Hiltner and J.R. Sites, Conference proceedings, 16th European Photovoltaic Solar Energy Conversion, Glasgow, 1-5 May, 2000, VB1.57, (File B154.pdf on CD version).

[4] S. Litvinenko, L. Ilchenko, and V. Skryshevsky, Materials Science and Engineering,. 71 (2000) 238.

[5] Kress, Pernau, T., Fath, P., and Bucher, E., (2000) LBIC measurements on low cost contact solar cells, Conference proceedings, 16th European Photovoltaic Solar Energy Conversion, Glasgow, 1-5 May, 2000, VA1.39, (File D339.pdf on CD version).

[6] NREL - Technology brief (Document: NREL/MK-336-21116, 8/96)

[7] D.L. King, J. A. Kratochvil, M. A. Quintana., and T. J. McMahon, Applications for infrared imaging equipment in photovoltaic cell, modules and system testing, WWW-Link: http://www.sandia.gov/pv/ieee2000/kingquin.pdf.

[8] M. Langenkamp, O. Breitenstein, M.E. Nell, H.-G. Wagemann, and L. Estner, Conference proceedings, 16th European Photovoltaic Solar Energy Conversion, Glasgow, 1-5 May, 2000, VD3.39 (File D411.pdf on CD version) WWW-Link: http://www.mpi-halle.mpg.de/ solar/.

[9] G. Färber, R.A. Bardos, K.R. McIntosh, C.B. Honsberg and A.B. Sproul, Conference proceedings of the 2nd World Conference and Exhibition on Photovoltaic Solar Energy Conversion, Vienna, Austria, 6 - 10 July 1998.

[10] J. Boudaden, A. Riviere, D. Ballutaud, J.-C. Muller, and R. Monna, Conference proceedings, 16th European Photovoltaic Solar Energy Conversion, Glasgow, 1-5 May, 2000, VD3.62, (File D432.pdf on CD version).

[11] C. Häßler, S. Thurm, W. Koch, D. Karg, G. Pensl, Conference proceedings of the 13th European Photovoltaic Solar Energy Conference, Nice, (1995) 1364.

[12] A.S.H. van der Heide, A. Schönecker, G.P. Wyers, and W.C. Sinke, Conference proceedings, 16th European Photovoltaic Solar Energy Conversion, Glasgow, 1-5 May, 2000, VA1.60 (File D359.pdf on CD version).

[13] E. Delahoy, and A. M. Payne, Determination of the internal series resistance of CIS and CIGS photovoltaic cell structures, Conference proceedings, 25th IEEE Photovoltaic Spec. Conf., (1996), p. 841.

[14] J.F. Hiltner and J. Sites, Local Photocurrent and Resistivity Measurements with Micron Resolution, Conference proceedings, 28th IEEE Photovoltaic Spec. Conf (2000), p. 543. 